\title{
Cardiovascular Protective Effects of Plant Polysaccharides: A Review
}

\author{
Xinli Dong ${ }^{1,2 \dagger}$, Mengze Zhou ${ }^{1 \dagger}$, Yehong $\mathrm{Li}^{1,2}$, Yuxin $\mathrm{Li}^{1,2}$, Hui $\mathrm{Ji}^{1,2 *}$ and Qinghua $\mathrm{Hu}{ }^{1,2 *}$ \\ ${ }^{1}$ State Key Laboratory of Natural Medicines, China Pharmaceutical University, Nanjing, China, ${ }^{2}$ School of Pharmacy, China \\ Pharmaceutical University, Nanjing, China
}

OPEN ACCESS

Edited by:

Jing Zhao,

University of Macau, China

Reviewed by:

Zunpeng Shu,

Guangdong Pharmaceutical

University, China

Ming Lu,

Nanjing Medical University, China

Guo Chen,

Jinan University, China

*Correspondence:

Qinghua Hu

huqh@cpu.edu.cn

Hui Ji

Huijicpu@163.com

${ }^{\dagger}$ These authors have contributed equally to this work

Specialty section:

This article was submitted to

Ethnopharmacology,

a section of the journal

Frontiers in Pharmacology

Received: 26 September 2021

Accepted: 25 October 2021

Published: 18 November 2021

Citation:

Dong X, Zhou M, Li Y, Li Y, Ji H and Hu Q (2021) Cardiovascular Protective

Effects of Plant Polysaccharides:

A Review.

Front. Pharmacol. 12:783641 doi: 10.3389/fphar.2021.783641
Cardiovascular disease is a kind of heart, brain, and blood vessel injury disease by the interaction of various pathological factors. The pathogenesis of cardiovascular disease is complex with various risk factors, including abnormally elevated blood pressure, glucose, and lipid metabolism disorders, atherosclerosis, thrombosis, etc. Plant polysaccharides are a special class of natural products derived from plant resources, which have the characteristics of wide sources, diverse biological activities, and low toxicity or side effects. Many studies have shown that plant polysaccharides improve cardiovascular diseases through various mechanisms such as anti-oxidative stress, restoring the metabolism of biological macromolecules, regulating the apoptosis cascade to reduce cell apoptosis, and inhibiting inflammatory signal pathways to alleviate inflammation. This article reviews the pharmacological effects and protective mechanisms of some plant polysaccharides in modulating the cardiovascular system, which is beneficial for developing more effective drugs with low side effects for management of cardiovascular diseases.

Keywords: plant polysaccharides, cardiovascular diseases, pharmacological effects, protective mechanisms, low side effects

\section{INTRODUCTION}

Cardiovascular disease (CVD) is a type of chronic non-infectious disease caused by circulatory system damage (Abe et al., 2017), with the characteristics of high incidence and large mortality. In recent years, owing to human lifestyle changes, the prevalence of CVD has been on an upward trend, and its fatality rate far exceeds that of cancer and other diseases. Statistically, more than two-fifths of deaths are attributed to CVD, in the rural and urban Chinese death population in 2016 (Ma et al., 2020), which makes CVD become the number one killer that affects human health. With the increasing understanding of the pathogenesis of CVD, the level of medical care of CVD has made great progress. Still, there are some shortcomings in the clinical treatment of CVD that remain to be resolved. In terms of drugs, most of small molecule chemicals commonly used in clinical treatment of CVD have many adverse reactions, insignificant efficacy, low patient compliance and other disadvantages. Consequently, in the process of seeking new drugs, plant polysaccharides with multiple targets, good biocompatibility and low toxicity have gradually become a hot spot in the research of anti-CVD drugs.

As a kind of natural macromolecule polymer extracted from various parts of plants, plant polysaccharides are composed of ten or more monosaccharides through polymerization with glycosidic linkages ( $\mathrm{Yu}$ et al., 2018). A large number of studies have shown that plant polysaccharides have various bioactivities such as anti-tumor, immunomodulation, antioxidant, radioprotection, hepatoprotection, anti-virus (Xie et al., 2016; Yu et al., 2018), which play an important role in regulating human physiological functions. More importantly, several studies have 
also shown other functions of plant polysaccharides such as antioxidant, anti-hyperglycemic, anti-hypertensive, antiatherosclerosis, anti-myocardial ischemia etc. (Zaporozhets and Besednova, 2016). These pharmacological effects provide a theoretical basis for plant polysaccharides to treat CVD. This article reviews reported mechanisms by which plant polysaccharides protect CVD from the perspective of multiple pharmacological effects.

\section{PROTECTIVE EFFECTS OF PLANT POLYSACCHARIDES ON CARDIOVASCULAR SYSTEM}

Globally, CVD is not only the leading cause of the decline in people's quality of life, but a primary reason for death. The pathogenesis of CVD is complicated, including glucose or lipid metabolism disorders, endothelial dysfunction, oxidative stress, and inflammation response. Till now, atherosclerosis, myocardial ischemia, abnormally elevated blood pressure, and thrombosis are recognized as the main risk factors for inducing CVD (Benjamin et al., 2019). Plant polysaccharides from natural sources play a cardiovascular protective effect by improving these series of risk factors.

\section{The Effect of Plant Polysaccharides on Hypertension}

Hypertension characterized by an uncontrolled increase in blood pressure leads to arteriosclerosis and myocardial injury, which has been regarded as one of the major factors to induce a series of refractory CVDs including coronary heart disease, cerebrovascular disease (stroke) and heart failure (Huang et al., 2013). The occurrence and development of hypertension is related to quite a few factors, among them, the dysfunction of endothelial and vascular smooth muscle is one of the primary causes of hypertension. Previous studies have shown that administration of low-molecular-weight fucoidan (LMWF) extracted from brown algae promoted the phosphorylation of endothelial nitric oxide synthase (eNOS) at Ser1177 and upregulated the eNOs/NO signal of vascular endothelial cells, which significantly improved the vasodilation disorder induced by endothelial dysfunction and robustly reduced basal hypertension in Goto-Kakizaki type 2 diabetic rats (Cui et al., 2014). Additionally, the subsequent findings by the research group suggested that LMWF also alleviated the hyperresponsiveness of vascular smooth muscle caused by diabetes and effectively improved diabetes induced hypertension. The effect of anti-vascular smooth muscle hyper-responsiveness of LMWF is mainly achieved by restoring the activity of antioxidant enzymes to inhibit the production of ROS, and inhibiting COX-2 to reduce the level of vasoconstrictor TXA2 in vascular smooth muscle (Liang et al., 2016). Consistently, mean arterial blood pressure in both normal blood pressure rats and hypertensive rats were appreciably lowered by white mulberry fruit polysaccharides, which is also related to the increase of the release of NO in vascular endothelial cells (Wang et al., 2019).
In this study, the production of $\mathrm{NO}$ may be related to the activation of intracellular $\mathrm{Ca}^{2+}$ signaling and PI3K/AKT signaling pathway. In addition, Astragalus polysaccharides treatment also reduced the mean pulmonary artery pressure in rats with monocrotaline-induced pulmonary arterial hypertension by activating eNOS/NO signaling pathway (Yuan et al., 2017). Interestingly, several studies have demonstrated that plant polysaccharides can also reduce high blood pressure by inhibiting angiotension-converting enzyme (ACE), including acidic polysaccharides from gastrodia rhizome (Lee et al., 2012), Chickpea water-soluble polysaccharide (Mokni Ghribi et al., 2015), Cymodocea nodosa sulfated polysaccharide (Kolsi et al., 2016), Momordica charantia polysaccharide (Tan and Gan, 2016), as well as water-soluble polysaccharides from Ephedra alata (Soua et al., 2020) and Almond and Pistachio (Sila et al., 2014). They can not only alleviate vasoconstriction by inhibiting the formation of angiotensin II, but also reduce metabolism of vasodilator bradykinin through the inhibition of kininase II, thereby dilating blood vessels and lowering blood pressure. Figure 1 summarizes plant polysaccharides with antihypertension activities.

\section{The Effect of Plant Polysaccharides on Atherosclerosis}

Atherosclerosis (AS) is a lipid-driven vascular inflammatory disease accompanied by gradually formation of vascular occlusive plaques and thrombus at the lesion site, which in turn induces CVDs including myocardial and cerebral infraction. Polysaccharides from Nitraria retusa fruits (Rjeibi et al., 2019) improved the atherosclerosis index (AI) of hyperlipidemia mice induced by TritonX-100 by reducing the level of serum triglycerides (TG), total cholesterol (TC), lowdensity lipoprotein cholesterol (LDL-C), and increasing the level of high-density lipoprotein cholesterol (HDL-C). Treatment with Gastrodia rhizomes crude and acidic polysaccharides (Kim et al., 2012) also markedly reduced the content of serum TC, LDL-C and AI of high-fat diet rats, but had no effect on serum TG and HDL-C levels. Furthermore, Enteromorpha prolifera polysaccharide (Tang et al., 2013; Guo et al., 2021), polysaccharides from Porphyra yezoensis (Qian et al., 2014) also reversed abnormal serum lipid concentrations in rats or hamsters with high-fat feeding, which is beneficial to alleviate atherogenesis. Fan et al. (Fan et al., 2013) found that the effect of Okra polysaccharide in lowering the serum lipid contents of obese mice was related to the regulation of the expression of lipid metabolism-related genes. Likewise, administration of polysaccharides from Rosae laevigatae fruits (Yu et al., 2013; Zhang et al., 2020), the sulfated polysaccharide from Ulva pertusa (Qi and Sheng, 2015; Li et al., 2020) and Ophiopogon polysaccharide (Wang et al., 2017) decreased blood lipids also by affecting the expression of these genes. Surprisingly, the Ophiopogon polysaccharide (Shi et al., 2016) also lowered the blood lipid level of hyperlipidemia mice by combining with the cholesterol metabolite bile acid then promoting the excretion of cholesterol through feces. Cyclocarya paliurus polysaccharide improved the blood lipid levels of hyperlipidemia rats by up- 


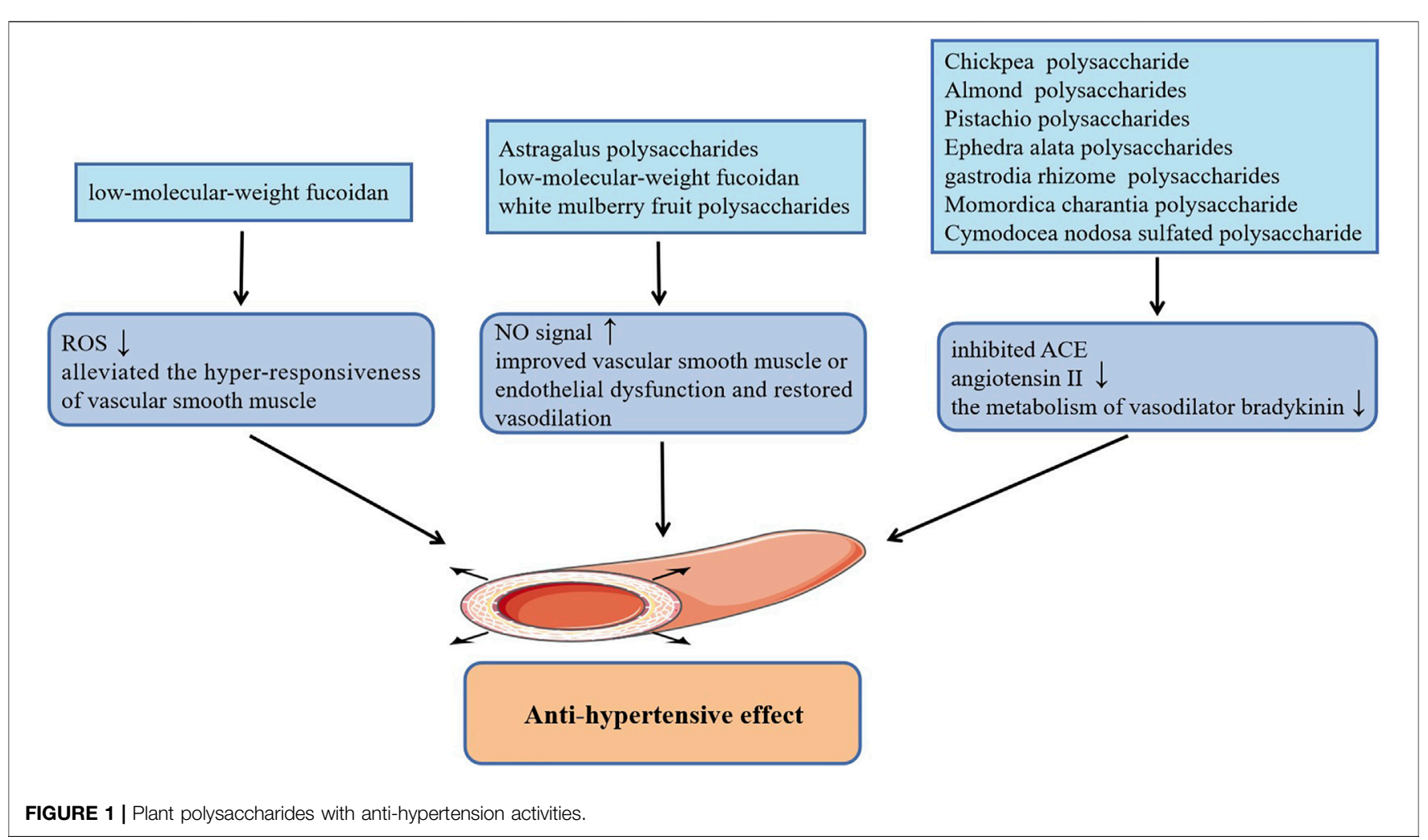

regulating the level of lipoprotein lipase, hormone-sensitive lipase as well as adipose triglyceride lipase, which promote lipid metabolism by down-regulating the level of acetyl-CoA carboxylase, fatty acid synthase as well as hydroxy methylglutaryl coenzyme A reductase (HMG-CoA) involved in lipid synthesis (Yang et al., 2016; Hu et al., 2017). Yang et al. also reported that Cyclocarya paliurus polysaccharide can regulate the expression of lipid metabolism enzymes by affecting the methylation level of related genes, thereby reducing blood lipids (Yang et al., 2019; Yang et al., 2021). Besides, fucoidan not only promoted lipid metabolism by regulating the expression of cholesterol metabolism-related genes, but inhibited the expression of aortic $\alpha$-smooth muscle actin ( $\alpha$-SMA), CD11b and vascular endothelial growth factor (VEGF), fibroblast growth factor-2 (FGF-2), P-SAPK as well as inflammatory cytokines, which alleviated atherosclerotic lesions in apolipoprotein E-deficient (apoE-/-) mice with high fat diet (Xu et al., 2019; Yin et al., 2019).

On the other hand, in atherosclerosis progression, macrophages can not only release inflammatory mediators to promote inflammatory response in the site of lesion, but excessively ingest lipids to transform into foam cells that are one of the components of atherosclerotic plaque. Remarkably, the administration of sulphated galactan isolated from the Acanthophora muscoides decreased the content of macrophages and tissue factor in the atherosclerotic plaques of apoE-/- mice with high-cholesterol diet by directly interferes with the chemotactic function of macrophages (GomesQuindere et al., 2015). In cholesterol crystals-pretreated macrophage-like THP-1 cells, treatment with Chayote polysaccharides reduced intracellular lipids levels by up-regulating the expression of liver $\mathrm{X}$ receptor alpha $(\operatorname{LXR} \alpha)$, and also inhibited the activation of inflammasome NLRP3 (Castro-Alves et al., 2019). Additionally, Red alga polysaccharides inhibited the activation of $\mathrm{NF}-\kappa \mathrm{B}$ and the up-regulation of intercellular vascular cell adhesion molecule-1 (VCAM-1) as well as adhesion molecule1 (ICAM-1) in human coronary artery endothelial cells (HCAECs) induced by angiotensin II (Hamias et al., 2018) or TNF- $\alpha$ (Levy-Ontman et al., 2017), which is helpful for alleviating inflammatory atherosclerosis progression. In addition, Opuntia dillenii Haw. Polysaccharides (Zhao et al., 2012) improved the aortic injury of hyperlipidemia rats by inhibiting the expression of VCAM-1 in the vascular endothelial and smooth muscle cells, which alleviated the process of AS. Figure 2 shows plant polysaccharides with anti-atherosclerosis actions.

\section{The Effect of Plant Polysaccharides on Thrombus}

Thrombus is a blood clot formed by the aggregation of insoluble fibrin, activated platelets, and other cells on the internal surface of blood vessels at the site of injury, including arterial thrombosis and venous thrombosis (Chan and Weitz, 2019). Several plant polysaccharides have the biological activity of anticoagulant and inhibiting platelet aggregation, which could effectively depress the thrombosis. Guar gum hydrolysate delayed the time to arterial blood flow decreases to zero, which is beneficial to depress arterial thrombosis induced by $\mathrm{Fecl}_{3}$ in hamster with high-fat diet (Kuo 


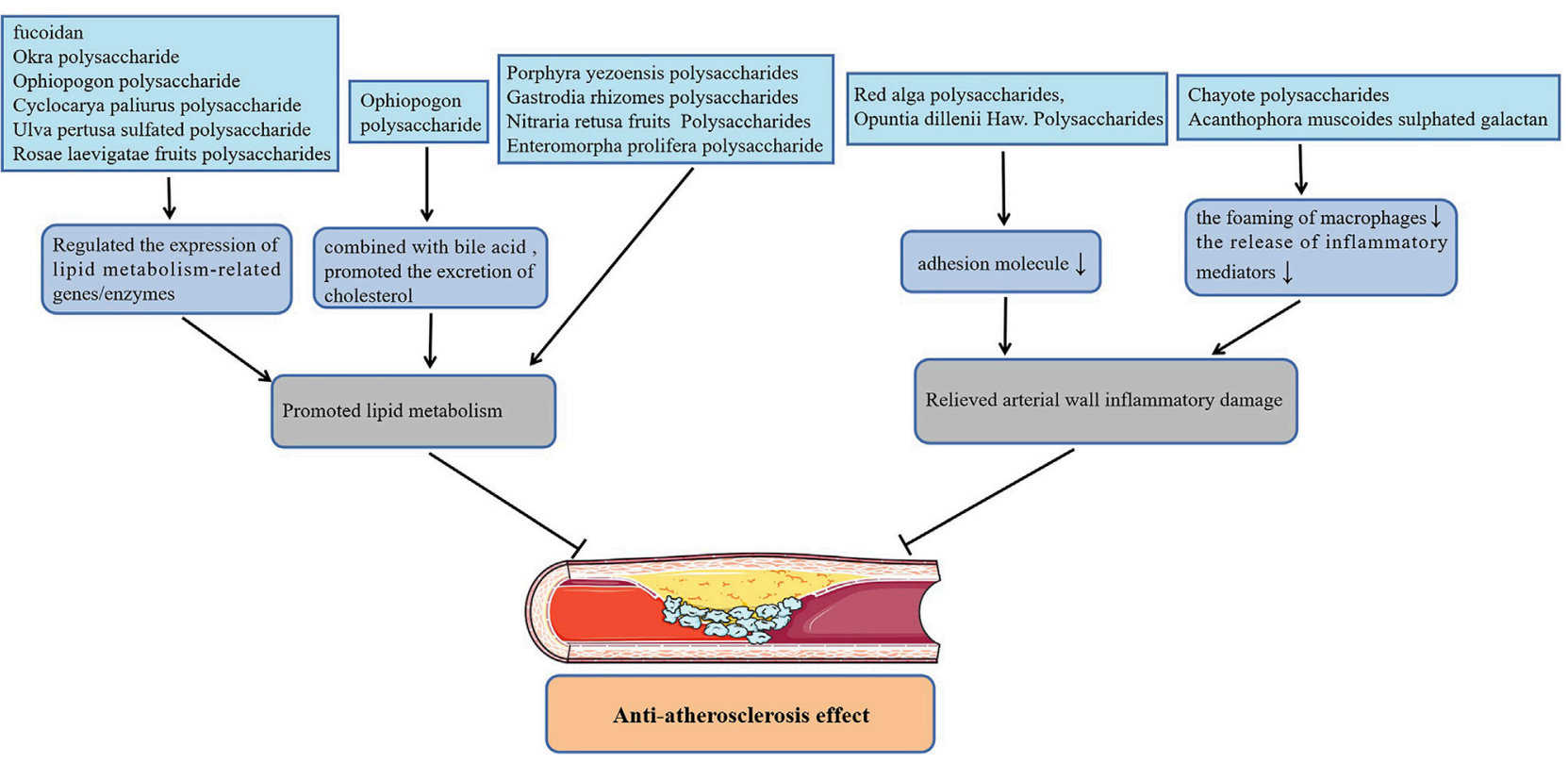

FIGURE 2 | Plant polysaccharides with anti-atherosclerosis actions.

et al., 2009). Consistently, chemically sulfated guar gum exhibited anticoagulant and antithrombotic effects in rats (de Oliveira Barddal et al., 2020). Similarly, sodium alginate sulfates inactivated $\alpha$-thrombin and coagulation factor Xa through the interaction between negative charges in the sulfate groups and the positively charges of anti-thrombin amino acid residues, exerting anticoagulant effect (Fan et al., 2011). Differently, sulfated Citrus pectin fractions inhibited coagulation factor $\mathrm{Xa}$ and platelet aggregation by directly inhibiting $\alpha$-thrombin, which attenuated venous thrombosis in rats (Cipriani et al., 2009). Additionally, sulfated rhamnan from Monostroma angicava (Liu D. et al., 2018), sulfated Pumpkin polysaccharide (Liang et al., 2018), sulfated Ginger polysaccharide (Wang et al., 2020), sulfated polysaccharides from Codium dwarkense børgesen (Golakiya et al., 2017), other sulfated polysaccharides extracted from seaweeds (Glauser et al., 2013; Chagas et al., 2020), as well as tea polysaccharides from Camellia sinensis (Cai et al., 2013) have been reported to have anticoagulant effects. On the other hand, Caesalpinia ferrea polysaccharides (de Araujo et al., 2021), polysaccharides of Geoffroea spinosa (Souza et al., 2015) and Lycium barbarum L. leaves polysaccharides (Lin et al., 2019) not only have anticoagulant activity but inhibit platelet aggregation, which exhibit depression effects on the formation of thrombus.

\section{The Effect of Plant Polysaccharides on Myocardial Ischemia and Myocardial Ischemia-Reperfusion Injury}

In recent years, persistent myocardial ischemia has becoming the primary cause of myocardial infarction (Thomes et al., 2010). As a classical approach, ischemia reperfusion could effectively restore the blood supply of ischemic myocardium, however, the production of a large amount of reactive oxygen species (ROS) and the infiltration of inflammatory cells caused by ischemia-reperfusion can also cause irreversible damage to the heart tissue (Hou et al., 2017). The biological activities of plant polysaccharides including anti-oxidant stress, anti-apoptosis, and anti-myocardial ischemia are beneficial to slow the progression of ischemic heart disease. Dendrobium officinale polysaccharides supplementation elevated serum SOD levels, up-regulated the expression of meis1, inhibited cardiomyocyte apoptosis, which significantly improved myocardial ischemic injury induced by coronary artery ligation in mice (Dou et al., 2016). Anti-oxidant and anti-apoptosis effects of Dendrobium officinale polysaccharide on cardiomyocytes were discovered using H9C2 cells damage model induced by $\mathrm{H}_{2} \mathrm{O}_{2}$ (Zhao et al., 2017). Ophiopogon japonicus polysaccharide promoted angiogenesis in myocardial ischemic tissue by activating SPHK/S1P/bFGF/AKT/ERK and eNOS/NO signaling pathways, which decreased the myocardial infarct size in rats with acute myocardial ischemia (Wang et al., 2012). It also increased endogenous antioxidants contents, $\mathrm{Na}^{+}-\mathrm{K}^{+}$-ATPase and $\mathrm{Ca}^{2+}-\mathrm{Mg}^{2+}$-ATPase activities in rats with isoproterenol (ISO)-induced myocardial ischemia (Fan et al., 2020). Momordica charantia polysaccharides protected rats against ISO-induced cardiomyocytes damage attributed to the depression of NF- $\kappa \mathrm{B}$, the increase of myocardial antioxidants levels and the decrease of pro-inflammatory factors (Raish, 2017). For rats with myocardial injury caused by cardiac ischemia or I/R, fucoidan plays a cardioprotective effect by improving oxidative stress, reducing the release of inflammatory factors and normalizing the $\mathrm{Na}^{+}-\mathrm{K}^{+}$-ATPase and $\mathrm{Ca}^{2+}-\mathrm{Mg}^{2+}$-ATPase levels (Li et al., 2011; Krishnamurthy et al., 2012). Notably, in cardiac I/R injury rats, Tamarind xyloglucan inhibited MAPK/bax/ caspase-3 apoptosis cascade by up-regulated the expression of 
fucoidan

Tamarind xyloglucan Larch arabinogalactan Soybean oligosaccharides Aralia elata polysaccharide Astragalus polysaccharides
Fructus aurantii polysaccharide Angelica sinensis polysaccharides Salvia miltiorrhiza polysaccharide Aloe vera selenium polysaccharides Momordica charantia polysaccharides Ophiopogon japonicus polysaccharide Dendrobium officinale polysaccharides

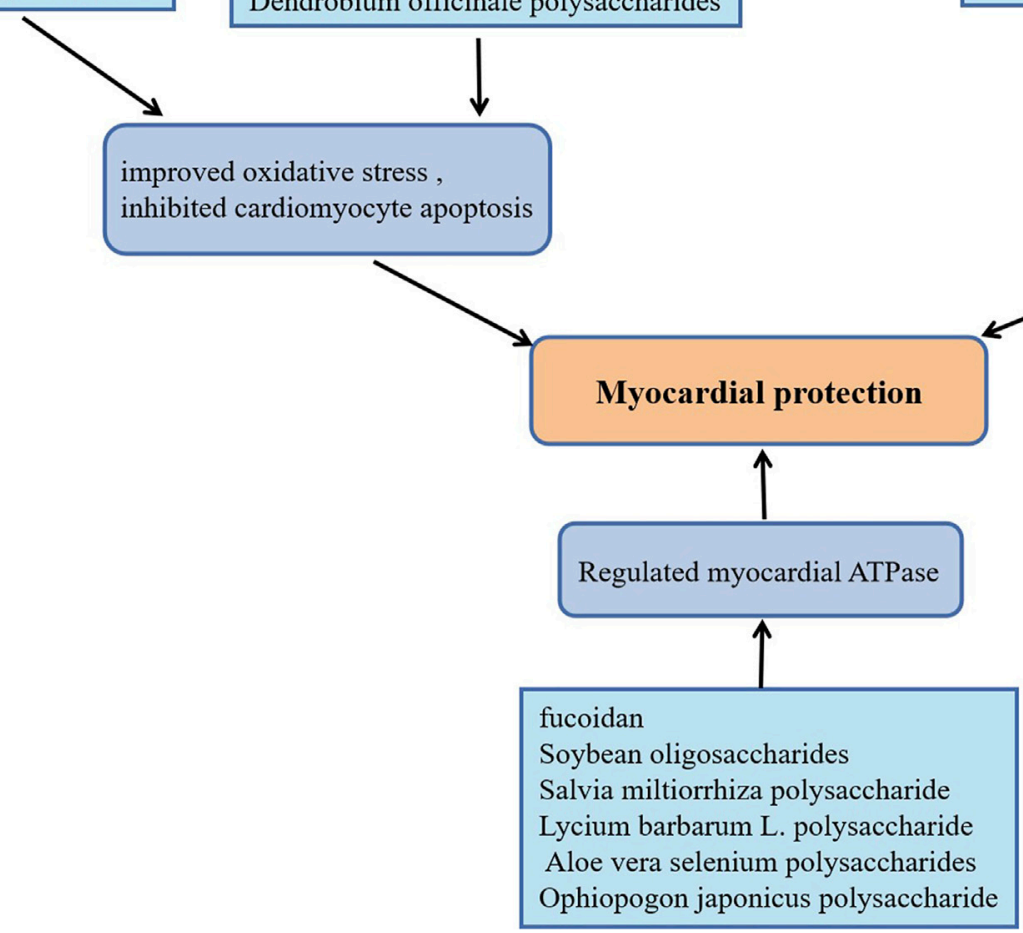

Ophiopogon japonicus polysaccharide

anti-myocardial ischemia

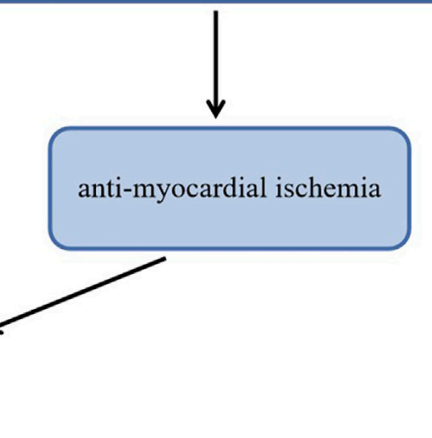

FIGURE 3 | Plant polysaccharides with myocardial protective effects.

fatty acid-binding protein (Lim and Lee, 2017), while Larch arabinogalactan depressed the cardiomyocytes apoptosis by inhibiting gelsolin/MAPK p38 and gelsolin/HIF-1 $\alpha$ signals, which effectively alleviated myocardial damage (Lim, 2017). Moreover, Astragalus polysaccharides (Liu X. et al., 2018), Angelica sinensis polysaccharides (Zhang et al., 2010), Aralia elata polysaccharide (Zhang et al., 2013), Aloe vera selenium polysaccharides (Yang et al., 2017), Salvia miltiorrhiza polysaccharide (Song et al., 2013; Geng et al., 2015) as well as Soybean oligosaccharides (Zhang et al., 2015) have been reported to the effects of anti-oxidation and reduce myocardial cell apoptosis in cardiac I/R model rats. More strikingly, Aloe vera selenium polysaccharides, Salvia miltiorrhiza polysaccharide and Soybean oligosaccharides also elevated the activities of $\mathrm{Na}^{+}-\mathrm{K}^{+}$ATPase and $\mathrm{Ca}^{2+}-\mathrm{Mg}^{2+}$-ATPase, which is consistent with the effect of Lycium barbarum L. polysaccharide on cardiomyocytes in cardiac I/R rats (Hou et al., 2017). In the experiment of hypoxia-reoxygenation treatment of $\mathrm{H} 9 \mathrm{C} 2$ cells, Fructus aurantii polysaccharide inhibited bax/caspase-mediated cells apoptosis and promoted the antioxidant effect mediated by Nrf2/HO-1 signal by activating the PI3K/AKT signaling pathway (Shu et al., 2020). Yang et al. also proved that Fructus aurantii polysaccharide has a protective effect on
ISO-induced myocardial ischemia injury in rats by exerting antioxidant and anti-apoptotic effects (Yang et al., 2020). Figure 3 exhibits plant polysaccharides with myocardial protective effects.

\section{DISCUSSION}

In recent years, with the rise of botanical medicine, the active ingredients in traditional herbs have gradually attracted people's attention. As one of the main active ingredients in most plant extracts, polysaccharides are widely used in research on the treatment of cardiovascular diseases. Nevertheless, most of the reports focus on the extraction, isolation, physical, and chemical properties of plant polysaccharides, but the pharmacological research of plant polysaccharides is relatively simple. We believe that the exact target of plant polysaccharides in vivo, and the cardiovascular protective mechanism at the molecular level need to be studied in depth in the future. On the other hand, although we generally accepted that plant polysaccharides had low toxicity or side effects, the structural uncertainty of plant polysaccharide monomers and individual differences might still lead to serious adverse events, so that the identification of plant 
polysaccharide molecular structure and adverse reactions clarification are necessary.

\section{AUTHOR CONTRIBUTIONS}

$\mathrm{XD}$ and $\mathrm{MZ}$ wrote the manuscript. YeL and $\mathrm{YuL}$ contributed to reviewing the manuscript. $\mathrm{QH}$ and $\mathrm{HJ}$ provided guidance on the

\section{REFERENCES}

Abe, H., Semba, H., and Takeda, N. (2017). The Roles of Hypoxia Signaling in the Pathogenesis of Cardiovascular Diseases. J. Atheroscler. Thromb. 24, 884-894. doi:10.5551/jat.RV17009

Benjamin, E. J., Muntner, P., Alonso, A., Bittencourt, M. S., Callaway, C. W., Carson, A. P., et al. (2019). Heart Disease and Stroke Statistics-2019 Update: A Report from the American Heart Association. Circulation 139, e56-e528. doi:10.1161/CIR.0000000000000659

Cai, W., Xie, L., Chen, Y., and Zhang, H. (2013). Purification, Characterization and Anticoagulant Activity of the Polysaccharides from green tea. Carbohydr. Polym. 92, 1086-1090. doi:10.1016/j.carbpol.2012.10.057

Castro-Alves, V. C., Shiga, T. M., and Nascimento, J. R. O. D. (2019). Polysaccharides from Chayote Enhance Lipid Efflux and Regulate NLRP3 Inflammasome Priming in Macrophage-like THP-1 Cells Exposed to Cholesterol Crystals. Int. J. Biol. Macromol 127, 502-510. doi:10.1016/ j.ijbiomac.2019.01.048

Chagas, F. D. D. S., Lima, G. C., Dos Santos, V. I. N., Costa, L. E. C., de Sousa, W. M., Sombra, V. G., et al. (2020). Sulfated Polysaccharide from the Red Algae Gelidiella Acerosa: Anticoagulant, Antiplatelet and Antithrombotic Effects. Int. J. Biol. Macromol 159, 415-421. doi:10.1016/j.ijbiomac.2020.05.012

Chan, N. C., and Weitz, J. I. (2019). Antithrombotic Agents. Circ. Res. 124, 426-436. doi:10.1161/circresaha.118.313155

Cipriani, T. R., Gracher, A. H., de Souza, L. M., Fonseca, R. J., Belmiro, C. L., Gorin, P. A., et al. (2009). Influence of Molecular Weight of Chemically Sulfated Citrus Pectin Fractions on Their Antithrombotic and Bleeding Effects. Thromb. Haemost. 101, 860-866. doi:10.1160/th08-08-0556

Cui, W., Zheng, Y., Zhang, Q., Wang, J., Wang, L., Yang, W., et al. (2014). Lowmolecular-weight Fucoidan Protects Endothelial Function and Ameliorates Basal Hypertension in Diabetic Goto-Kakizaki Rats. Lab. Invest. 94, 382-393. doi:10.1038/labinvest.2014.12

de Araujo, D. F., Madeira, J. D. C., Cunha, A. P., Ricardo, N. M. P. S., Bezerra, F. F., Mourão, P. A. S., et al. (2021). Structural Characterization of Anticoagulant and Antithrombotic Polysaccharides Isolated from Caesalpinia Ferrea Stem Barks. Int. J. Biol. Macromol 175, 147-155. doi:10.1016/j.ijbiomac.2021.01.177

de Oliveira Barddal, H. P., Faria, F. A. M., Nogueira, A. V., Iacomini, M., and Cipriani, T. R. (2020). Anticoagulant and Antithrombotic Effects of Chemically Sulfated Guar Gum. Int. J. Biol. Macromol 145, 604-610. doi:10.1016/ j.ijbiomac.2019.12.210

Dou, M. M., Zhang, Z. H., Li, Z. B., Zhang, J., and Zhao, X. Y. (2016). Cardioprotective potential of Dendrobium officinale Kimura et Migo against myocardial ischemia in mice. Mol. Med. Rep. 14, 4407-4414. doi:10.3892/ mmr.2016.5789

Fan, L., Jiang, L., Xu, Y., Zhou, Y., Shen, Y., Xie, W., et al. (2011). Synthesis and Anticoagulant Activity of Sodium Alginate Sulfates. Carbohydr. Polym. 83, 1797-1803. doi:10.1016/j.carbpol.2010.10.038

Fan, S., Guo, L., Zhang, Y., Sun, Q., Yang, B., and Huang, C. (2013). Okra Polysaccharide Improves Metabolic Disorders in High-Fat Diet-Induced Obese C57BL/6 Mice. Mol. Nutr. Food Res. 57, 2075-2078. doi:10.1002/ mnfr.201300054

Fan, S., Zhang, J., Xiao, Q., Liu, P., Zhang, Y., Yao, E., et al. (2020). Cardioprotective Effect of the Polysaccharide from Ophiopogon Japonicus on IsoproterenolInduced Myocardial Ischemia in Rats. Int. J. Biol. Macromol 147, 233-240. doi:10.1016/j.ijbiomac.2020.01.068

Geng, Z. H., Huang, L., Song, M. B., and Song, Y. M. (2015). Protective Effect of a Polysaccharide from Salvia Miltiorrhiza on Isoproterenol (ISO)-induced framework of the review. All authors contributed to the editing and submission of the review.

\section{FUNDING}

This research was supported by Natural Science Foundation of China (Grants 81773745, 81872867).

Myocardial Injury in Rats. Carbohydr. Polym. 132, 638-642. doi:10.1016/ j.carbpol.2015.06.086

Glauser, B. F., Mourão, P. A., and Pomin, V. H. (2013). Marine Sulfated Glycans with Serpin-Unrelated Anticoagulant Properties. Adv. Clin. Chem. 62, 269-303. doi:10.1016/b978-0-12-800096-0.00007-x

Golakiya, H. N., Naik, V. N., Hirapara, H. N., Mody, K. H., Goswami, A. P., and Tripathi, C. (2017). Evaluation of Anticoagulant Effect of Sulfated Polysaccharide (Sps) from Codium Dwarkense Borgesen in $\kappa$-Carrageenan Induced Hypercoagulable State in Wistar Albino Rats. Acta Pol. Pharm. 74, 987-994.

Gomes Quinderé, A. L., Barros Benevides, N. M., Pelli, G., Lenglet, S., Burger, F., Carbone, F., et al. (2015). Treatment with Sulphated Galactan Inhibits Macrophage Chemotaxis and Reduces Intraplaque Macrophage Content in Atherosclerotic Mice. Vascul Pharmacol. 71, 84-92. doi:10.1016/ j.vph.2015.02.015

Guo, F., Han, M., Lin, S., Ye, H., Chen, J., Zhu, H., et al. (2021). Enteromorpha Prolifera Polysaccharide Prevents High- Fat Diet-Induced Obesity in Hamsters: A NMR-Based Metabolomic Evaluation. J. Food Sci. 86, 3672-3685. doi:10.1111/1750-3841.15818

Hamias, R., Wolak, T., Huleihel, M., Paran, E., and Levy-Ontman, O. (2018). Red Alga Polysaccharides Attenuate Angiotensin II-Induced Inflammation in Coronary Endothelial Cells. Biochem. Biophys. Res. Commun. 500, 944-951. doi:10.1016/j.bbrc.2018.04.206

Hou, Y. M., Wang, J., and Zhang, X. Z. (2017). Lycium Barbarum Polysaccharide Exhibits Cardioprotection in an Experimental Model of Ischemia-Reperfusion Damage. Mol. Med. Rep. 15, 2653-2658. doi:10.3892/mmr.2017.6294

Hu, W.-B., Zhao, J., Chen, H., Xiong, L., and Wang, W.-J. (2017). Polysaccharides from Cyclocarya Paliurus: Chemical Composition and Lipid-Lowering Effect on Rats Challenged with High-Fat Diet. J. Funct. Foods 36, 262-273. doi:10.1016/j.jff.2017.07.020

Huang, W. Y., Davidge, S. T., and Wu, J. (2013). Bioactive Natural Constituents from Food Sources-Potential Use in Hypertension Prevention and Treatment. Crit. Rev. Food Sci. Nutr. 53, 615-630. doi:10.1080/ 10408398.2010.550071

Kim, K. J., Lee, O. H., Han, C. K., Kim, Y. C., and Hong, H. D. (2012). Acidic Polysaccharide Extracts from Gastrodia Rhizomes Suppress the Atherosclerosis Risk index through Inhibition of the Serum Cholesterol Composition in Sprague Dawley Rats Fed a High-Fat Diet. Int. J. Mol. Sci. 13, 1620-1631. doi:10.3390/ijms13021620

Kolsi, R. B. A., Fakhfakh, J., Krichen, F., Jribi, I., Chiarore, A., Patti, F. P., et al (2016). Structural Characterization and Functional Properties of Antihypertensive Cymodocea Nodosa Sulfated Polysaccharide. Carbohydr. Polym. 151, 511-522. doi:10.1016/j.carbpol.2016.05.098

Krishnamurthy, M., Selvaraju, M., and Tamilarasan, M. (2012). Turbinaria Conoides (J. Agardh) Sulfated Polysaccharide Protects Rat's Heart against Myocardial Injury. Int. J. Biol. Macromol 50, 1275-1279. doi:10.1016/ j.ijbiomac.2012.03.012

Kuo, D. C., Hsu, S. P., and Chien, C. T. (2009). Partially Hydrolyzed Guar Gum Supplement Reduces High-Fat Diet Increased Blood Lipids and Oxidative Stress and Ameliorates FeCl3-Induced Acute Arterial Injury in Hamsters. J. Biomed. Sci. 16, 15. doi:10.1186/1423-0127-16-15

Lee, O. H., Kim, K. I., Han, C. K., Kim, Y. C., and Hong, H. D. (2012). Effects of Acidic Polysaccharides from Gastrodia Rhizome on Systolic Blood Pressure and Serum Lipid Concentrations in Spontaneously Hypertensive Rats Fed a HighFat Diet. Int. J. Mol. Sci. 13, 698-709. doi:10.3390/ijms13010698

Levy-Ontman, O., Huleihel, M., Hamias, R., Wolak, T., and Paran, E. (2017). An Anti-inflammatory Effect of Red Microalga Polysaccharides in Coronary Artery 
Endothelial Cells. Atherosclerosis 264, 11-18. doi:10.1016/ j.atherosclerosis.2017.07.017

Li, B., Xu, H., Wang, X., Wan, Y., Jiang, N., Qi, H., et al. (2020). Antioxidant and Antihyperlipidemic Activities of High Sulfate Content Purified Polysaccharide from Ulva Pertusa. Int. J. Biol. Macromol 146, 756-762. doi:10.1016/ j.ijbiomac.2019.11.061

Li, C., Gao, Y., Xing, Y., Zhu, H., Shen, J., and Tian, J. (2011). Fucoidan, a Sulfated Polysaccharide from Brown Algae, against Myocardial Ischemia-Reperfusion Injury in Rats via Regulating the Inflammation Response. Food Chem. Toxicol. 49, 2090-2095. doi:10.1016/j.fct.2011.05.022

Liang, L., Ao, L., Ma, T., Ni, Y., Liao, X., Hu, X., et al. (2018). Sulfated Modification and Anticoagulant Activity of Pumpkin (Cucurbita Pepo, Lady Godiva) Polysaccharide. Int. J. Biol. Macromol 106, 447-455. doi:10.1016/ j.ijbiomac.2017.08.035

Liang, Z., Zheng, Y., Wang, J., Zhang, Q., Ren, S., Liu, T., et al. (2016). Low Molecular Weight Fucoidan Ameliorates Streptozotocin-Induced HyperResponsiveness of Aortic Smooth Muscles in Type 1 Diabetes Rats. J. Ethnopharmacol 191, 341-349. doi:10.1016/j.jep.2016.06.054

Lim, S. H. (2017). Larch Arabinogalactan Attenuates Myocardial Injury by Inhibiting Apoptotic Cascades in a Rat Model of Ischemia-Reperfusion. J. Med. Food 20, 691-699. doi:10.1089/jmf.2016.3886

Lim, S. H., and Lee, J. (2017). Xyloglucan Intake Attenuates Myocardial Injury by Inhibiting Apoptosis and Improving Energy Metabolism in a Rat Model of Myocardial Infarction. Nutr. Res. 45, 19-29. doi:10.1016/j.nutres.2017.07.003

Lin, S., Al-Wraikat, M., Niu, L., Zhou, F., Zhang, Y., Wang, M., et al. (2019). Degradation Enhances the Anticoagulant and Antiplatelet Activities of Polysaccharides from Lycium Barbarum L. Leaves. Int. J. Biol. Macromol 133, 674-682. doi:10.1016/j.ijbiomac.2019.04.147

Liu, D., Chen, L., Zhao, J., and Cui, K. (2018a). Cardioprotection Activity and Mechanism of Astragalus Polysaccharide In Vivo and In Vitro. Int. J. Biol. Macromol 111, 947-952. doi:10.1016/j.ijbiomac.2018.01.048

Liu, X., Wang, S., Cao, S., He, X., Qin, L., He, M., et al. (2018b). Structural Characteristics and Anticoagulant Property In Vitro and In Vivo of a Seaweed Sulfated Rhamnan. Mar. Drugs 16, 243. doi:10.3390/md16070243

Ma, L. Y., Chen, W. W., Gao, R. L., Liu, L. S., Zhu, M. L., Wang, Y. J., et al. (2020). China Cardiovascular Diseases Report 2018: an Updated Summary. J. Geriatr. Cardiol. 17, 1-8. doi:10.11909/j.issn.1671-5411.2020.01.001

Mokni Ghribi, A., Sila, A., Maklouf Gafsi, I., Blecker, C., Danthine, S., Attia, H., et al. (2015). Structural, Functional, and ACE Inhibitory Properties of WaterSoluble Polysaccharides from Chickpea Flours. Int. J. Biol. Macromol 75, 276-282. doi:10.1016/j.ijbiomac.2015.01.037

Qi, H., and Sheng, J. (2015). The Antihyperlipidemic Mechanism of High Sulfate Content Ulvan in Rats. Mar. Drugs 13, 3407-3421. doi:10.3390/md13063407

Qian, L., Zhou, Y., and Ma, J. X. (2014). Hypolipidemic Effect of the Polysaccharides from Porphyra Yezoensis. Int. J. Biol. Macromol 68, 48-49. doi:10.1016/j.ijbiomac.2014.04.004

Raish, M. (2017). Momordica Charantia Polysaccharides Ameliorate Oxidative Stress, Hyperlipidemia, Inflammation, and Apoptosis during Myocardial Infarction by Inhibiting the NF-Kb Signaling Pathway. Int. J. Biol. Macromol 97, 544-551. doi:10.1016/j.ijbiomac.2017.01.074

Rjeibi, I., Feriani, A., Hentati, F., Hfaiedh, N., Michaud, P., and Pierre, G. (2019). Structural Characterization of Water-Soluble Polysaccharides from Nitraria Retusa Fruits and Their Antioxidant and Hypolipidemic Activities. Int. J. Biol. Macromol 129, 422-432. doi:10.1016/j.ijbiomac.2019.02.049

Shi, L., Wang, J., Wang, Y., and Feng, Y. (2016). MDG-1, an Ophiopogon Polysaccharide, Alleviates Hyperlipidemia in Mice Based on Metabolic Profile of Bile Acids. Carbohydr. Polym. 150, 74-81. doi:10.1016/ j.carbpol.2016.05.008

Shu, Z., Yang, Y., Ding, Z., Wang, W., Zhong, R., Xia, T., et al. (2020). Structural Characterization and Cardioprotective Activity of a Novel Polysaccharide from Fructus Aurantii. Int. J. Biol. Macromol 144, 847-856. doi:10.1016/ j.ijbiomac.2019.09.162

Sila, A., Bayar, N., Ghazala, I., Bougatef, A., Ellouz-Ghorbel, R., and EllouzChaabouni, S. (2014). Water-soluble Polysaccharides from Agro-Industrial By-Products: Functional and Biological Properties. Int. J. Biol. Macromol 69, 236-243. doi:10.1016/j.ijbiomac.2014.05.052

Song, M., Huang, L., Zhao, G., and Song, Y. (2013). Beneficial Effects of a Polysaccharide from Salvia Miltiorrhiza on Myocardial Ischemia-
Reperfusion Injury in Rats. Carbohydr. Polym. 98, 1631-1636. doi:10.1016/ j.carbpol.2013.08.020

Soua, L., Koubaa, M., Barba, F. J., Fakhfakh, J., Ghamgui, H. K., and Chaabouni, S. E. (2020). Water-Soluble Polysaccharides from Ephedra Alata Stems: Structural Characterization, Functional Properties, and Antioxidant Activity. Molecules 25, 2210. doi:10.3390/molecules25092210

Souza, R. O., Assreuy, A. M., Madeira, J. C., Chagas, F. D., Parreiras, L. A., Santos, G. R., et al. (2015). Purified Polysaccharides of Geoffroea Spinosa Barks Have Anticoagulant and Antithrombotic Activities Devoid of Hemorrhagic Risks. Carbohydr. Polym. 124, 208-215. doi:10.1016/j.carbpol.2015.01.069

Tan, H. F., and Gan, C. Y. (2016). Polysaccharide with Antioxidant, $\alpha$-amylase Inhibitory and ACE Inhibitory Activities from Momordica Charantia. Int. J. Biol. Macromol 85, 487-496. doi:10.1016/j.ijbiomac.2016.01.023

Tang, Z., Gao, H., Wang, S., Wen, S., and Qin, S. (2013). Hypolipidemic and Antioxidant Properties of a Polysaccharide Fraction from Enteromorpha Prolifera. Int. J. Biol. Macromol 58, 186-189. doi:10.1016/ j.ijbiomac.2013.03.048

Thomes, P., Rajendran, M., Pasanban, B., and Rengasamy, R. (2010). Cardioprotective Activity of Cladosiphon Okamuranus Fucoidan against Isoproterenol Induced Myocardial Infarction in Rats. Phytomedicine 18 (1), 52-57. doi:10.1016/j.phymed.2010.06.006

Wang, C., Cheng, W., Bai, S., Ye, L., Du, J., Zhong, M., et al. (2019). White mulberry Fruit Polysaccharides Enhance Endothelial Nitric Oxide Production to Relax Arteries In Vitro and Reduce Blood Pressure In Vivo. Biomed. Pharmacother. 116, 109022-022. doi:10.1016/j.biopha.2019.109022

Wang, C., He, Y., Tang, X., and Li, N. (2020). Sulfation, Structural Analysis, and Anticoagulant Bioactivity of Ginger Polysaccharides. J. Food Sci. 85, 2427-2434. doi:10.1111/1750-3841.15338

Wang, S., Lin, X., Wang, L. Y., Ruan, K. F., Feng, Y., and Li, X. Y. (2012). A Polysaccharides MDG-1 Augments Survival in the Ischemic Heart by Inducing S1P Release and S1P1 Expression. Int. J. Biol. Macromol 50, 734-740. doi:10.1016/j.ijbiomac.2011.12.005

Wang, X., Shi, L., Joyce, S., Wang, Y., and Feng, Y. (2017). MDG-1, a Potential Regulator of PPARa and PPAR $\gamma$, Ameliorates Dyslipidemia in Mice. Int. J. Mol. Sci. 18, 1930. doi:10.3390/ijms18091930

Xie, J. H., Jin, M. L., Morris, G. A., Zha, X. Q., Chen, H. Q., Yi, Y., et al. (2016). Advances on Bioactive Polysaccharides from Medicinal Plants. Crit. Rev. Food Sci. Nutr. 56 (Suppl. 1), S60-S84. doi:10.1080/10408398.2015.1069255

Xu, Y., Zhu, W., Wang, T., Jin, L., Liu, T., Li, X., et al. (2019). Low Molecule Weight Fucoidan Mitigates Atherosclerosis in ApoE (-/-) Mouse Model through Activating Multiple Signal Pathway. Carbohydr. Polym. 206, 110-120. doi:10.1016/j.carbpol.2018.10.097

Yang, Y., Ding, Z., Zhong, R., Xia, T., Wang, W., Zhao, H., et al. (2020). Cardioprotective Effects of a Fructus Aurantii Polysaccharide in Isoproterenol-Induced Myocardial Ischemic Rats. Int. J. Biol. Macromol 155, 995-1002. doi:10.1016/j.ijbiomac.2019.11.063

Yang, Y., Yang, M., Ai, F., and Huang, C. (2017). Cardioprotective Effect of Aloe Vera Biomacromolecules Conjugated with Selenium Trace Element on Myocardial Ischemia-Reperfusion Injury in Rats. Biol. Trace Elem. Res. 177, 345-352. doi:10.1007/s12011-016-0896-8

Yang, Z., Zhao, J., Li, J., Wang, J., and Wang, W. (2021). Genome-wide DNA Methylation Profiling of High-Fat Emulsion-Induced Hyperlipidemia Mice Intervened by a Polysaccharide from Cyclocarya Paliurus (Batal) Iljinskaja. Food Chem. Toxicol. 152, 112230. doi:10.1016/j.fct.2021.112230

Yang, Z., Zhao, J., Wang, J., Li, J., Ouyang, K., and Wang, W. (2019). Effects of Cyclocarya Paliurus Polysaccharide on Lipid Metabolism-Related Genes DNA Methylation in Rats. Int. J. Biol. Macromol 123, 343-349. doi:10.1016/ j.ijbiomac.2018.11.110

Yang, Z. W., Ouyang, K. H., Zhao, J., Chen, H., Xiong, L., and Wang, W. J. (2016). Structural Characterization and Hypolipidemic Effect of Cyclocarya Paliurus Polysaccharide in Rat. Int. J. Biol. Macromol 91, 1073-1080. doi:10.1016/ j.ijbiomac.2016.06.063

Yin, J., Wang, J., Li, F., Yang, Z., Yang, X., Sun, W., et al. (2019). The Fucoidan from the Brown Seaweed Ascophyllum Nodosum Ameliorates Atherosclerosis in Apolipoprotein E-Deficient Mice. Food Funct. 10, 5124-5139. doi:10.1039/ c9fo00619b

Yu, C. H., Dai, X. Y., Chen, Q., Zang, J. N., Deng, L. L., Liu, Y. H., et al. (2013). Hypolipidemic and Antioxidant Activities of Polysaccharides from Rosae 
Laevigatae Fructus in Rats. Carbohydr. Polym. 94, 56-62. doi:10.1016/ j.carbpol.2013.01.006

Yu, Y., Shen, M., Song, Q., and Xie, J. (2018). Biological Activities and Pharmaceutical Applications of Polysaccharide from Natural Resources: A Review. Carbohydr. Polym. 183, 91-101. doi:10.1016/j.carbpol.2017.12.009

Yuan, L. B., Hua, C. Y., Gao, S., Yin, Y. L., Dai, M., Meng, H. Y., et al. (2017). Astragalus Polysaccharides Attenuate Monocrotaline-Induced Pulmonary Arterial Hypertension in Rats. Am. J. Chin. Med. 45, 773-789. doi:10.1142/ S0192415X17500410

Zaporozhets, T., and Besednova, N. (2016). Prospects for the Therapeutic Application of Sulfated Polysaccharides of Brown Algae in Diseases of the Cardiovascular System: Review. Pharm. Biol. 54, 3126-3135. doi:10.1080/ 13880209.2016.1185444

Zhang, J., Wang, H., and Zheng, Q. (2013). Cardioprotective Effect of Aralia Elata Polysaccharide on Myocardial Ischemic Reperfusion (IR) Injury in Rats. Int. J. Biol. Macromol 59, 328-332. doi:10.1016/ j.ijbiomac.2013.04.060

Zhang, M., Cai, S., and Ma, J. (2015). Evaluation of Cardio-Protective Effect of Soybean Oligosaccharides. Gene 555, 329-334. doi:10.1016/j.gene.2014.11.027

Zhang, S., He, B., Ge, J., Li, H., Luo, X., Zhang, H., et al. (2010). Extraction, Chemical Analysis of Angelica Sinensis Polysaccharides and Antioxidant Activity of the Polysaccharides in Ischemia-Reperfusion Rats. Int. J. Biol. Macromol 47, 546-550. doi:10.1016/j.ijbiomac.2010.07.012

Zhang, X., Hu, Y., Jin, C., and Wu, W. (2020). Extraction and Hypolipidemic Activity of Low Molecular Weight Polysaccharides Isolated from Rosa Laevigata Fruits. Biomed. Res. Int. 2020, 2043785. doi:10.1155/2020/2043785
Zhao, L. Y., Huang, W., Yuan, Q. X., Cheng, J., Huang, Z. C., Ouyang, L. J., et al. (2012) Hypolipidaemic Effects and Mechanisms of the Main Component of Opuntia Dillenii Haw. Polysaccharides in High-Fat Emulsion-Induced Hyperlipidaemic Rats. Food Chem. 134, 964-971. doi:10.1016/j.foodchem.2012.03.001

Zhao, X., Dou, M., Zhang, Z., Zhang, D., and Huang, C. (2017). Protective Effect of Dendrobium Officinale Polysaccharides on H2O2-Induced Injury in H9c2 Cardiomyocytes. Biomed. Pharmacother. 94, 72-78. doi:10.1016/ j.biopha.2017.07.096

Conflict of Interest: The authors declare that the research was conducted in the absence of any commercial or financial relationships that could be construed as a potential conflict of interest.

Publisher's Note: All claims expressed in this article are solely those of the authors and do not necessarily represent those of their affiliated organizations, or those of the publisher, the editors and the reviewers. Any product that may be evaluated in this article, or claim that may be made by its manufacturer, is not guaranteed or endorsed by the publisher.

Copyright (C) 2021 Dong, Zhou, Li, Li, Ji and Hu. This is an open-access article distributed under the terms of the Creative Commons Attribution License (CC BY). The use, distribution or reproduction in other forums is permitted, provided the original author(s) and the copyright owner(s) are credited and that the original publication in this journal is cited, in accordance with accepted academic practice. No use, distribution or reproduction is permitted which does not comply with these terms. 